\title{
Asociación bidireccional entre uso de tabaco y riesgo de depresión en el estudio de cohorte SUN
}

\author{
Bidirectional association between tobacco use and \\ depression risk in the SUN cohort study
}

\author{
Almudena Sánchez-Villegas*,**, Alfredo Gea***, Francisca Lahortiga-Ramos****, Julio \\ Martínez-González*****, Patricio Molero****, Miguel A. Martínez-González**,***,******. \\ * Grupo de Investigación en Nutrición, Instituto Universitario de Investigaciones Biomédicas y Sanitarias, Universidad de Las \\ Palmas de Gran Canaria, Las Palmas de Gran Canaria, España. \\ ** Ciber de Fisiopatología de la Obesidad y Nutrición (CIBER OBN), Instituto de Salud Carlos III, Madrid, España. \\ *** Universidad de Navarra, Departamento de Medicina Preventiva y Salud Pública, Pamplona, España. \\ **** Departamento de Psiquiatría y Psicología Médica, Clínica Universidad de Navarra, Pamplona, España. \\ ***** Hospital Carlos Haya, Departamento de Medicina Interna, Málaga, España. \\ ****** Departamento de Nutrición, Escuela de Salud Pública TH Chan de la Universidad de Harvard, Boston, EE. UU.
}

\section{Resumen}

Evaluamos la asociación del consumo de tabaco o su abandono con el riesgo de depresión y determinamos si presentar depresión se asoció al inicio de fumar. Diseño: estudio de cohortes prospectivo de 16.519 graduados universitarios españoles sin depresión al inicio del estudio. El consumo de tabaco se determinó al inicio y tras cuatro años de seguimiento. Los casos incidentes de depresión fueron autoinformados en los cuestionarios de seguimiento. Usamos modelos de regresión de Cox para estimar los Hazard Ratios (HR) de depresión según el nivel de tabaquismo inicial y sus cambios. Se estimaron los Odds Ratios (OR) para la asociación entre prevalencia de tabaquismo a lo largo de la vida y comienzo del hábito con modelos de regresión logística. Se observó un HR (IC 95\%) para fumadores de 1,24 (1,05-1,46) en comparación con los que nunca habían fumado. Los participantes con la exposición más alta al tabaco ( $\geq 20$ paquetes-año) tuvieron un incremento relativo del riesgo de depresión de $38 \%$. Dejar de fumar durante los primeros cuatro años de seguimiento se asoció inversamente con la depresión ( $\mathrm{HR}=0,63$; IC 95\% = 0,40-0,99). Observamos un incremento significativo del riesgo de aparición de tabaquismo en los participantes con prevalencia de depresión a lo largo de la vida (OR multivariable = 1,44; IC $95 \%=1,13-1,83)$. Encontramos una asociación bidireccional entre el consumo de tabaco y la depresión en la cohorte SUN. El control del tabaco y las campañas sanitarias de abstinencia deberían considerarse estrategias efectivas de salud pública para prevenir y manejar los trastornos depresivos.

Palabras clave: Fumar; paquetes-año; cohorte; riesgo de depresión; dejar de fumar; comienzo de fumar.

\section{Abstract}

We assessed the association of tobacco use or smoking cessation with depression risk and determined if the presence of a depressive disorder was associated with smoking onset. We conducted a prospective cohort study (SUN Project) based on 16,519 Spanish university graduates without depression at baseline. Tobacco use was determined at baseline and after four years of follow-up. Incident cases of depression were ascertained according to a previously validated report of a clinical diagnosis of depression during follow-up. Multivariable Cox regression models were used to estimate hazard ratios (HR) of depression according to previous smoking status. We used logistic regression models as a secondary analysis to estimate Odds Ratios (OR) of smoking onset during the first four years of follow-up according to lifetime depression prevalence at baseline. The multivariable HR (95\% CI) for current smokers was 1.24 (1.05-1.46) as compared to participants who had never smoked. Participants with the highest exposure to tobacco $(\geq 20$ packs-years) had a significant $38 \%$ relative increment in depression risk. Smoking cessation during the first four years of follow-up was inversely associated with depression $(\mathrm{HR}=0.63 ; 95 \% \mathrm{CI}=0.40-0.99)$. Finally, a significant increment in the risk of smoking onset for participants with lifetime depression prevalence was observed (multivariable OR = $1.44 ; 95 \% \mathrm{CI}=1.13-1.83$ ). A bidirectional association between tobacco use and depression in the SUN cohort was found. Therefore, tobacco control and health promotion campaigns for smoking cessation could be considered as effective strategies of public health for the prevention and management of depressive disorders.

Key words: Smoking; packs-year; cohort; depression risk; smoking cessation; smoking onset.

Enviar correspondencia a: Francisca Lahortiga-Ramos y Patricio Molero. Dpto. Psiquiatría y Psicología Médica, Clínica Universidad de Navarra, Avda. Pio XII 36, 31008 Pamplona, España.

E-mail: flahortiga@unav.es; pmolero@unav.es 
L a depresión unipolar se considera una causa global importante de años de vida ajustados en función de la discapacidad (AVAD) (Kyu et al., 2018) y esta enfermedad fue la tercera causa de años de vida perdidos (AVP) por discapacidad en 2017 (James et al., 2018). En este contexto, la prevención de la depresión es un objetivo importante desde una perspectiva de salud pública. La depresión es una enfermedad multifactorial. Varios factores de estilo de vida han sido analizados como posibles determinantes modificables de desarrollo de la depresión. La dieta (Lassale et al., 2019; Opie et al., 2017), la actividad física (Fernández-Montero et al., 2020) y los hábitos tóxicos (Kedzior y Laeber, 2014) son determinantes clínicamente relevantes y modificables del riesgo de depresión; los hábitos tóxicos se refieren al tabaquismo, la ingesta de alcohol y el uso de drogas ilícitas. Respecto del tabaco, una revisión sistemática ha sugerido una asociación bidireccional entre el tabaquismo y la salud mental medida como depresión y ansiedad, donde el tabaquismo aumenta el riesgo de enfermedades mentales y las enfermedades mentales aumentan el riesgo de inicio de tabaquismo (Fluharty, Taylor, Grabski y Munafò, 2017). Es más, otro metaanálisis arrojó reducciones significativas en la sintomatología depresiva después del cese del tabaquismo (Taylor et al., 2014). Algunos autores también han observado una tendencia hacia una asociación lineal entre el número de cigarrillos/día consumidos y la gravedad de la depresión según la escala de evaluación para la depresión de Hamilton (HDRS), lo que sugiere una relación entre la gravedad de consumo y la gravedad de los síntomas de depresión (Jiménez-Treviño et al., 2019). Por último, varios estudios han informado de que las personas con enfermedad mental tienden a ser más propensas a adoptar hábitos tabáquicos y de fumar en mayor medida que la población general (Fluharty et al., 2017; Fluharty, Sallis y Munafò, 2018). En este contexto, escasean los estudios epidemiológicos longitudinales que hayan hecho un análisis simultáneo de todos estos aspectos. Consideramos que nuestro estudio aporta una aclaración para determinar la asociación entre el hábito tabáquico, incluido el uso, la dosis, la duración del tabaquismo y del abandono del tabaquismo, y el riesgo de desarrollar depresión, así como analizar si tener un trastorno depresivo representa o no un factor de riesgo para el inicio/recaída de tabaquismo utilizando un diseño epidemiológico prospectivo. El uso de este tipo de diseño nos permitió evaluar con eficiencia tanto el papel de la depresión en el inicio o la recaída del tabaquismo como el papel y la duración del consumo de tabaco en el riesgo de desarrollar depresión después de varios años de seguimiento.

Por tanto, este estudio tuvo como objetivos (1) evaluar la asociación entre el tabaco (el uso, la dosis y la duración medidos en paquetes-año de consumo de cigarrillos) y el riesgo de depresión; (2) evaluar el papel del cese del taba- quismo en el riesgo de depresión; (3) y, por último, determinar si la presencia de un trastorno depresivo se asoció con el inicio del tabaquismo en el estudio de cohorte SUN.

\section{Método}

\section{Población de estudio}

El Proyecto SUN es un estudio de cohorte dinámico y prospectivo, iniciado en diciembre de 1999 en España. La muestra está compuesta por titulados universitarios españoles que participan voluntariamente. Sus métodos han sido descritos previamente (Carlos et al., 2018; Martínez-González, Sánchez-Villegas, De Irala, Marti y Martínez, 2002). En resumen, los datos de características sociodemográficas y de estilo de vida, las variables antropométricas y el historial médico o el uso de medicamentos se recopila bienalmente por correo o mediante cuestionarios basados en la web enviados a los participantes cada dos años. La retención general en la cohorte se aproxima al $90 \%$.

Hasta julio de 2018, 22.791 participantes habían completado el cuestionario de referencia del Proyecto SUN. Fueron excluidos del análisis los participantes que se perdieron durante el seguimiento, que no habían completado al menos un cuestionario de seguimiento o que estaban fuera de los límites predefinidos para la ingesta total de energía al inicio del estudio. Para los análisis que consideraban la incidencia de depresión como resultado, también se excluyó a los participantes que tomaban antidepresivos o que habían informado al inicio del estudio de un historial actual o previo de diagnóstico clínico de depresión (depresión a lo largo de la vida). Finalmente, se incluyeron 16.519 participantes en los análisis prospectivos para la incidencia de depresión de nueva aparición (Figura 1). En un análisis complementario, seleccionamos a 11.246 participantes de la cohorte con al menos cuatro años de seguimiento y que eran no fumadores o exfumadores al inicio del estudio para evaluar el papel de la prevalencia de por vida de la depresión en la recaída. (Figura 2).

El estudio fue aprobado por la Junta Institucional de Revisión de la Universidad de Navarra y registrado en clinicaltrials.gov (NCT02669602). No se solicitó a los participantes el consentimiento informado por escrito al considerar que la cumplimentación voluntaria del primer cuestionario implicó consentimiento informado.

\section{Evaluación de la exposición}

El consumo de tabaco se determinó al inicio del estudio y después de cuatro años de seguimiento. Un participante se consideró como fumador actual o exfumador en caso de responder afirmativamente a la pregunta: «¿Ha fumado 100 o más cigarrillos a lo largo de su vida?» en el cuestionario de referencia (Q_0). A los participantes que respondieron de manera positiva se les preguntó además sobre el número medio de cigarrillos consumidos a diario 
en algunos períodos específicos de su vida. Si un participante refirió ser exfumador, se le preguntó cuánto tiempo hacía desde que dejó de fumar. Después, se clasificaron a los exfumadores en dos grupos: exfumadores que habían dejado de fumar hace diez o más años y exfumadores que habían dejado de fumar hace menos de 10 años. Este punto de corte fue elegido porque se consideró que diez años es un periodo relevante para determinar la superación del tabaquismo y asegurar que la condición del participante es realmente la de exfumador.

También se estimó el número de paquetes-año consumidos por fumadores actuales y exfumadores. Usamos la definición de paquetes-año según el Diccionario de Términos de Cáncer del Instituto Nacional del Cáncer; se calcularon paquetes-año multiplicando el número de paquetes de cigarrillos fumados por día por el número de años que la persona había fumado. Por ejemplo, 1 paquete-año es igual a fumar 1 paquete por día durante 1 año o 2 paquetes por día durante medio año, y así sucesivamente (Instituto Nacional del Cáncer, 2019).
Los participantes actualizan su consumo de tabaco tras cuatro años de seguimiento. De esta manera se determinó el número de personas que dejaron o empezaron a fumar.

\section{Resultados de la evaluación}

Los casos incidentes de depresión se definieron como participantes que respondieron de forma positiva a la pregunta «¿Alguna vez un médico le ha diagnosticado depresión?» en cualquiera de los cuestionarios bienales de seguimiento a partir del segundo año de seguimiento (Q_2-Q_16). El autoinforme de un diagnóstico médico de depresión ha mostrado una validez aceptable en una submuestra de nuestra cohorte utilizando la Entrevista Clínica Estructurada para el DSM-IV como instrumento de referencia aplicado por psiquiatras experimentados «cegados» a las respuestas de los cuestionarios (Sánchez-Villegas et al., 2008a). El porcentaje de depresión confirmada fue 74,2\% [IC 95\% (IC) = 63,3-85,1]. El porcentaje de depresión no confirmada fue $81,1 \%$ (IC $95 \%=69,1-92,9)$.

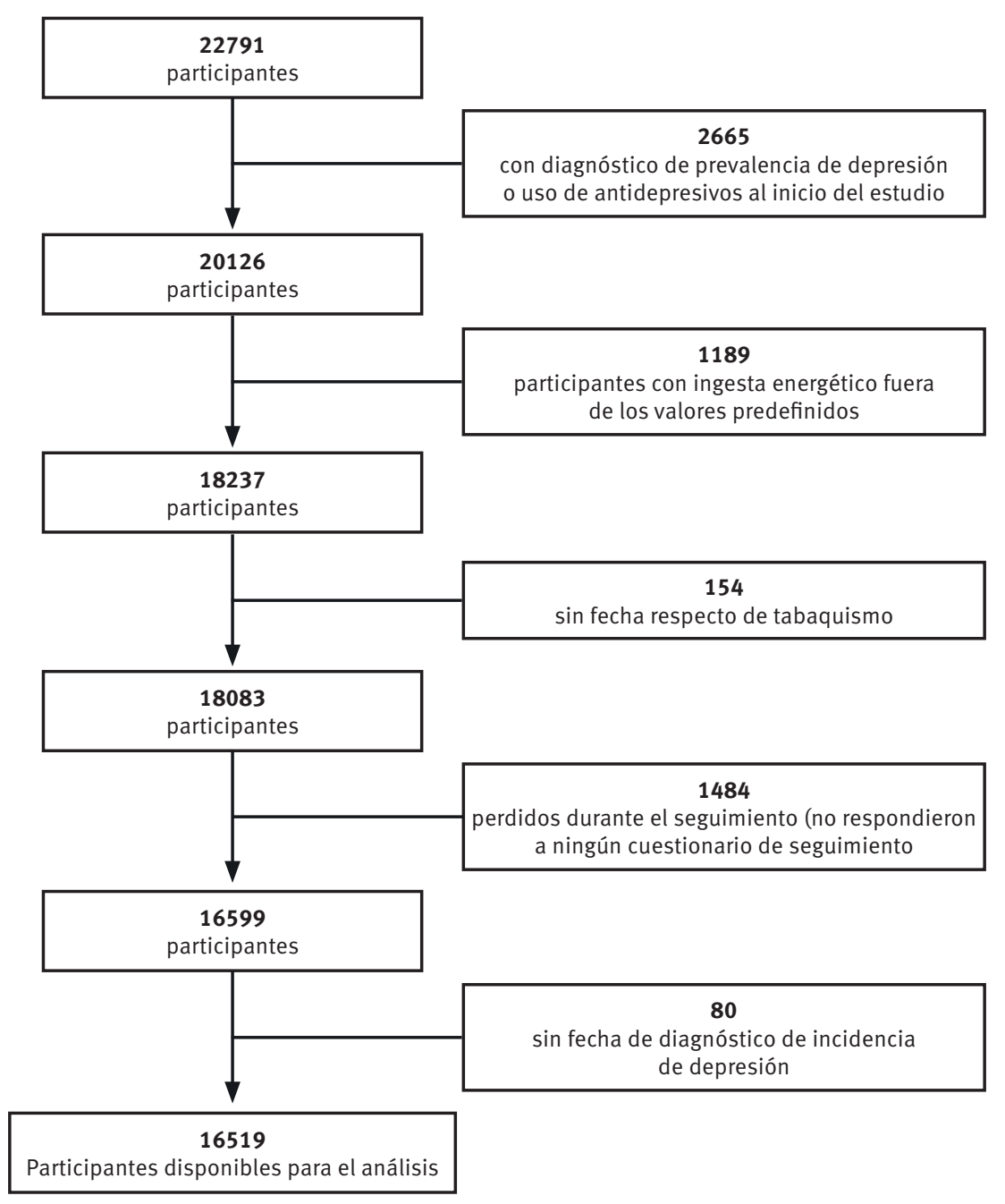

Figura 1. Diagrama de flujo para la selección de participantes. 


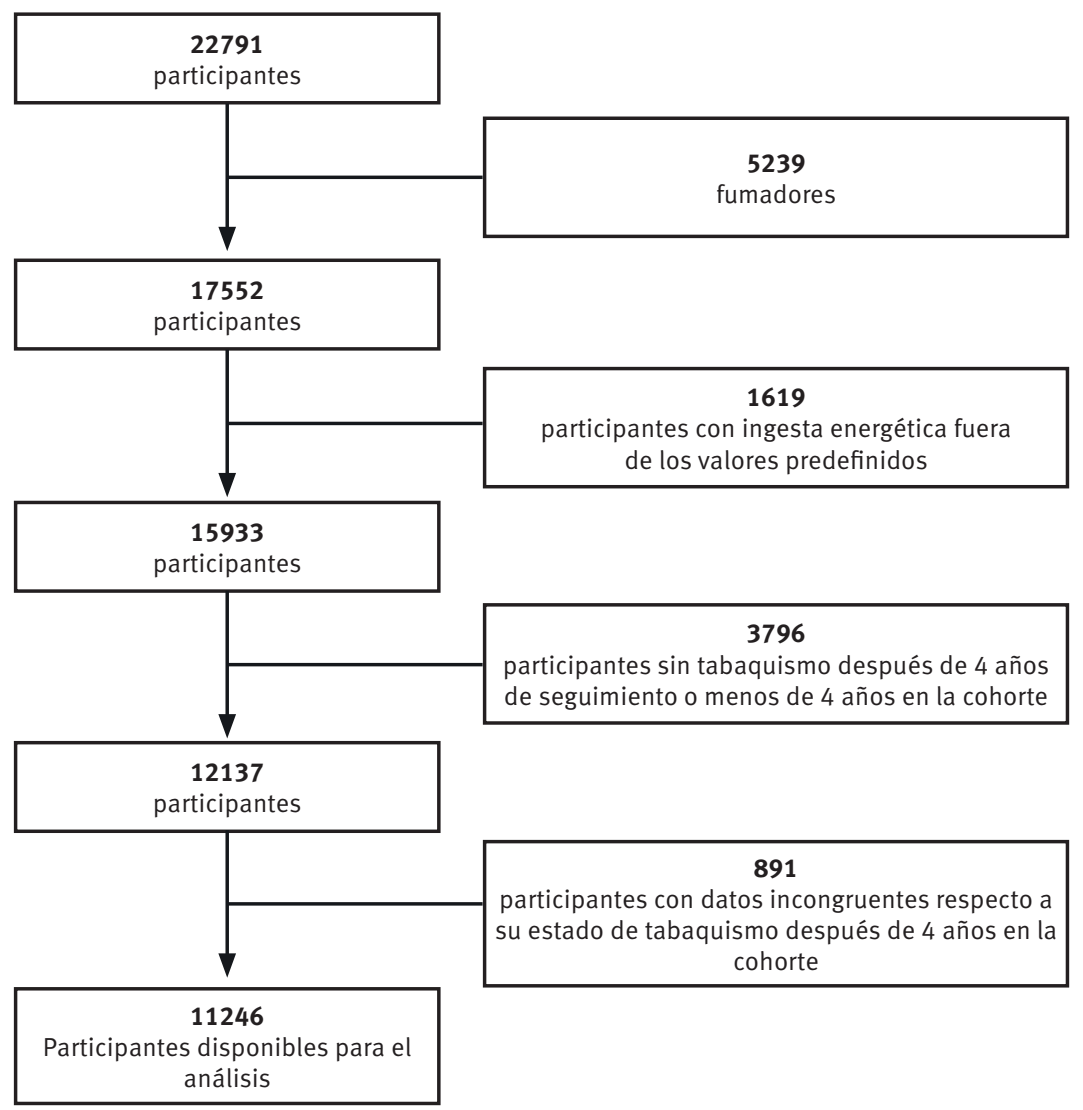

Figura 2. Diagrama de flujo para la selección de participantes (análisis secundario).

\section{Evaluación de otras variables}

La información sobre variables sociodemográficas ( $p$. ej., sexo, edad, estado civil y situación laboral) y relacionadas con el estilo de vida (p. ej., actividad física, hábitos alimentarios) se obtuvo del Q_0. La actividad física se evaluó mediante un cuestionario validado de actividad física con datos sobre diecisiete actividades utilizadas en poblaciones de habla hispana y previamente adaptado de los EE. UU. (Nurses' Health Study and Health Professionals' Follow-up Study) (Martínez-González, López-Fontana, Varo, Sánchez-Villegas y Martínez, 2005). El estudio de validación calculó los coeficientes de correlación no paramétricos entre el nivel de actividad física y el estilo de vida sedentario recogidos por el cuestionario autoadministrado y las mediciones de un acelerómetro triaxial. También se calcularon el porcentaje de clasificación errónea y los coeficientes kappa. La población de estudio consistió en una muestra de 40 mujeres con obesidad, participantes del Proyecto SUN. La actividad física durante el tiempo de ocio (estimada como MET-h semana) derivado del cuestionario autoadministrado tuvo una correlación moderada con las kcal/día evaluadas con el uso del acelerómetro $(r$ de Spearman $=$ 0,507, IC 95\% 0,232-0,707). La correlación de Spearman de la relación entre estilo de vida sedentario y actividad física obtenida a través del cuestionario y la estimación directa
(RT3) fue de -0,578 (IC 95\% -0,754, -0,325). El índice kappa fue de $0,25(p=, 002)$ al evaluar la clasificación cruzada en quintiles y 0,41 para la estimación dicotómica de estilo de vida sedentario. Solo el 2,5\% de los participantes fueron clasificados erróneamente por el cuestionario en más de dos quintiles de distancia de las estimaciones del RT3.

La ingesta dietética se evaluó al inicio del estudio con el cuestionario validado y semicuantitativo de frecuencia de consumo de alimentos (FFQ) de 136 ítems (De La Fuente-Arrillaga, Vázquez-Ruiz, Bes-Rastrollo, Sampson y Martínez-González, 2010; Fernández-Ballart et al., 2010). La ingesta de nutrientes y energía se calculó mediante la multiplicación de la frecuencia por la composición nutricional del tamaño de la porción especificada para cada alimento utilizando un programa informático ad hoc desarrollado específicamente para este fin. Un dietista entrenado actualizó la base de datos de nutrientes utilizando la información más reciente disponible incluida en las tablas de composición de alimentos para España (Mataix, 2003; Moreiras, Carbajal, Cabrera y Cuadrado, 2005). La adherencia a la dieta mediterránea se evaluó utilizando la información incluida en el FFQ y calculando la puntuación MEDAS (Schröder et al., 2011).

La información sobre los rasgos de personalidad (nivel autopercibido de competitividad, tensión psicológica 
y dependencia) también se obtuvo con el Q_0 utilizando escalas Likert. Los participantes fueron clasificados en tres grupos diferentes de acuerdo a sus respuestas en el Q_0: nivel bajo (1-4), nivel moderado (5-6) y nivel alto (7-10).

El índice de masa corporal (IMC) se calculó como peso (kg) dividido por altura cuadrada $\left(\mathrm{m}^{2}\right)$ con los datos recopilados en el Q_0. La validez del peso y la talla autoinformados se evaluó en la cohorte (Bes-Rastrollo, Pérez-Valdivieso, Sánchez-Villegas, Alonso y Martínez-González, 2005).

La prevalencia y los antecedentes de cáncer, enfermedades cardiovasculares, hipertensión y diabetes mellitus tipo 2 se determinaron al inicio del estudio. La enfermedad cardiovascular incluye infarto de miocardio, accidente cerebrovascular, fibrilación auricular, taquicardia paroxística, cirugía de revascularización coronaria u otros procedimientos de revascularización, insuficiencia cardíaca, aneurisma aórtico, embolia pulmonar o trombosis venosa periférica. Todos los diagnósticos se basaron en autoinformes de los participantes. La validez del diagnóstico autoinformado de hipertensión se evaluó en una submuestra de la cohorte (Alonso, Beunza, Delgado-Rodríguez y Martínez-González, 2005).

\section{Métodos estadísticos}

Las características de los participantes al inicio del estudio ajustados por edad y sexo, utilizando la ponderación de probabilidad inversa, se describieron según su hábito de fumar utilizando frecuencias relativas, medias y desviaciones estándar.

Para cada participante, se calculó persona-años de seguimiento desde la fecha de entrega del Q_0 hasta la primera de dos fechas: diagnóstico de depresión o entrega del último cuestionario de seguimiento.

Se ajustaron modelos de regresión de riesgos proporcionales de Cox para evaluar la relación entre el consumo de tabaco y la incidencia de depresión durante el seguimiento. Se calcularon los Hazard Ratios (HR) y su IC de 95\% con los no fumadores como categoría de referencia. Para controlar los posibles factores de confusión, realizamos un modelo ajustado multivariable que ajusta por sexo, edad, IMC $\left(\mathrm{kg} / \mathrm{m}^{2}\right.$, continuo), actividad física durante el tiempo de ocio (METs/h-w, continuo), ingesta total de energía ( $\mathrm{kcal} / \mathrm{d}$, continuo), adherencia a la dieta mediterránea (114, continuo), ingesta de alcohol (abstemios; 0.1-9.9 g/d; 10-25 g/d; > $25 \mathrm{~g} / \mathrm{d}$ ), vivir solo/a (sí, no, desconocido), desempleado/a (sí/no), años de educación (continuo) y rasgos de personalidad (tres categorías: competitividad, tensión psicológica, dependencia). Otros factores de confusión, tales como la presencia de varias enfermedades (cáncer, enfermedad cardiovascular, hipertensión y diabetes) o el estado civil, también se exploraron, pero fueron omitidos de los modelos finales porque su inclusión en los modelos de regresión no cambió de manera importante las asociaciones reportadas.
El papel del cese del tabaquismo (durante los primeros cuatro años de seguimiento) en el riesgo de depresión también se evaluó mediante modelos de regresión multivariables de riesgos proporcionales de Cox, con el no cese del tabaquismo como valor de referencia. Representamos la incidencia acumulada ajustada de depresión según el hábito tabáquico, utilizando la ponderación de probabilidad inversa. Luego, estimamos el número necesario a tratar (NNT) como el inverso de la diferencia de riesgo, que se estimó mediante un modelo logístico agrupado. El IC de $95 \%$ era los percentiles 2,5 y 97,5 de la distribución obtenida de un bootstrap no paramétrico con 1000 muestras.

Los análisis de subgrupos se realizaron según sexo, edad, adherencia a la dieta mediterránea, nivel de actividad física y rasgos de personalidad como variables de estratificación. Para evaluar las posibles interacciones se introdujeron términos de producto en los diferentes modelos multivariable. Los valores $p$ para la interacción se calcularon utilizando la prueba de razón de verosimilitud.

Además, se realizaron varios análisis de sensibilidad tras la (1) consideración de un tiempo máximo de seguimiento de 6 años (los participantes con un período de seguimiento más largo fueron censurados a 6 años y los casos de depresión ocurridos después del sexto año se consideraron no depresión); (2) consideración solo de los casos de depresión diagnosticados después de 2 años de seguimiento; (3) consideración solo de los casos de depresión diagnosticados después de 4 años de seguimiento y (4) exclusión de los participantes con cáncer, enfermedad cardiovascular o diabetes tipo 2 al inicio del estudio.

Entre los exfumadores, también se calculó la posible asociación no lineal entre el número de años desde el cese del tabaquismo y el riesgo de depresión con el mismo método. Las pruebas de no linealidad utilizaron la prueba de razón de verosimilitud, comparando el modelo solo con el término lineal con el modelo con splines cúbicos. Los resultados se ajustaron para los mismos factores de confusión potenciales que el análisis de regresión de Cox principal.

Por último, para evaluar una posible asociación bidireccional tabaco-depresión, analizamos el papel de la depresión en el inicio del tabaquismo. En un análisis auxiliar basado en 11.246 participantes de la cohorte (con al menos cuatro años de seguimiento y siendo no fumadores o exfumadores al inicio del estudio), analizamos la asociación entre la prevalencia de depresión a lo largo de la vida al inicio del estudio y la probabilidad de inicio/recaída de tabaquismo después de cuatro años de seguimiento en la cohorte, mediante el uso de modelos de regresión logística ajustados multivariables con el inicio del tabaquismo durante los primeros 4 años de seguimiento como resultado. Los análisis se repitieron después de separar en dos grupos diferentes a los que nunca habían fumado y los exfumadores y analizar el papel de la depresión en el inicio del tabaquismo y la recaída del tabaquismo de forma individual. 
Todos los valores $p$ fueron bilaterales y se consideró significativo $p<, 05$. El análisis estadístico se realizó utilizando STATA versión 12.0 (StataCorp).

\section{Resultados}

La tabla 1 muestra las características de los participantes al inicio del estudio según las categorías de tabaquismo. Los fumadores actuales eran más jóvenes que los exfumadores, tenían más probabilidades de estar solteros, y tendían a tener un menor nivel de actividad física, una mayor ingesta de alcohol y menor adherencia a la dieta mediterránea. Sin embargo, la prevalencia de enfermedades crónicas importantes al inicio del estudio (incluido el cáncer, enfermedad cardiovascular, diabetes o hipertensión) fue menor entre estos participantes.

$\mathrm{Al}$ tratarse de una cohorte dinámica, el tiempo de seguimiento no es igual para todos los participantes incluidos en el análisis. El tiempo máximo de seguimiento fue de aproximadamente 13,5 años; sin embargo, la mediana de seguimiento en este análisis fue de 11,1 años.

Para el análisis principal, durante un total de 174.754 persona-año de seguimiento, encontramos 889 casos incidentes de depresión. La tabla 2 muestra la asociación entre el tabaquismo y la depresión. Los resultados indican una asociación directa y significativa entre el tabaquismo y el riesgo de depresión. El HR multivariable (IC 95\%) para la depresión en los fumadores actuales era de 1,24 (1,05$1,46)$ en comparación con los que nunca habían fumado. No encontramos ninguna asociación significativa para los exfumadores independientemente de los años desde que dejaron de fumar en comparación con los que nunca habían fumado. Sin embargo, en comparación con los fumadores actuales, ser exfumador se asoció con una menor incidencia de depresión: 0,85 (0,70-1,02). Esta asociación fue especialmente relevante para los fumadores que dejaron de fumar más de 10 años antes del inicio del estudio: 0,74 (0,57-0,97).

Cuando se repitió el análisis separando a los que nunca habían fumado y los exfumadores, la asociación entre la presencia de depresión al inicio del estudio y el riesgo de inicio/recaída del tabaquismo dejó de ser significativa. El OR multivariable para la asociación entre la prevalencia de depresión a lo largo de la vida y el inicio del tabaquismo entre los que nunca habían fumado era 1,13 (0,71-1,82), mientras que el OR para la recaída del tabaquismo entre los exfumadores era de 1,26 (0,89-1,78).

La dosis de tabaco y la duración del tabaquismo también tuvieron una asociación directa con el riesgo de depresión. Los participantes con una mayor exposición a los productos de tabaco o una duración prolongada del tabaquismo (20 o más paquetes-año) tuvieron un incremento significativo del $38 \%$ en el riesgo relativo de desarrollar depresión durante el seguimiento (IC 95\% = 1,07-1,79). Además, el abandono del tabaquismo durante los primeros cuatro años de seguimiento se asoció inversamente con el riesgo de depresión ( $\mathrm{HR}=0,63$; IC 95\% =0,40-0,99) en comparación con los fumadores actuales. Como muestra la figura 3, el riesgo de los participantes que dejaron de fumar alcanza las tasas de los que nunca habían fumado después de apro-

Tabla 1. Características al inicio del estudio de los participantes en el estudio de cohorte SUN, según tabaquismo ajustado para edad y sexo con la ponderación de probabilidad inversa.

\begin{tabular}{|c|c|c|c|c|}
\hline & $\begin{array}{l}\text { Nunca ha sido fumador } \\
\qquad(\mathrm{n}=8022)\end{array}$ & $\begin{array}{l}\text { Exfumador }>10 a^{*} \\
\qquad(\mathrm{n}=1854)\end{array}$ & $\begin{array}{l}\text { Exfumador }<10 a^{*} \\
\quad(n=2674)\end{array}$ & $\begin{array}{l}\text { Fumador actual } \\
\qquad(\mathrm{n}=3510)\end{array}$ \\
\hline Índice de masa corporal $\left(\mathrm{kg} / \mathrm{m}^{2}\right)$ & $23,5(3,6)$ & $24,5(3,6)$ & $23,9(3,6)$ & $23,5(3,5)$ \\
\hline Actividad física (MET-h/w) & $24,2(23,1)$ & $23,9(23,7)$ & $22,3(21,4)$ & $20,3(19,6)$ \\
\hline Ingesta de energía (kcal/día) & $2345(625)$ & $2308(617)$ & $2320(611)$ & 2347 (629) \\
\hline Dieta mediterránea (1-14) & $6,0(2,0)$ & $6,3(1,9)$ & $5,9(1,9)$ & $5,7(1,8)$ \\
\hline Ingesta de alcohol (g/día) & $4,9(7,5)$ & $8,7(12,2)$ & $8,2(11,6)$ & $8,9(12,2)$ \\
\hline Nivel de competitividad (0-10) & $7,0(1,7)$ & $6,9(1,8)$ & $6,9(1,7)$ & $6,9(1,8)$ \\
\hline Tensión psicológica (0-10) & $5,8(2,3)$ & $6,0(2,1)$ & $6,0(2,2)$ & $5,8(2,3)$ \\
\hline Nivel de dependencia (0-10) & $3,5(2,8)$ & $3,5(3,0)$ & $3,7(2,9)$ & $3,6(2,9)$ \\
\hline Años de estudios universitarios & $5,1(1,6)$ & $5,3(1,6)$ & $5,0(1,4)$ & $5,0(1,4)$ \\
\hline Vive solo/a (\%) & 7,0 & 4,1 & 7,5 & 6,9 \\
\hline Desempleado/a (\%) & 3,9 & 2,4 & 3,5 & 4,5 \\
\hline Casado/a (\%) & 48,8 & 77,4 & 57,0 & 48,2 \\
\hline \multicolumn{5}{|l|}{ Prevalencia de enfermedad (\%) } \\
\hline Cáncer & 3,5 & 5,1 & 3,3 & 3,0 \\
\hline Enfermedad cardiovascular & 1,7 & 1,9 & 2,2 & 1,1 \\
\hline Diabetes & 2,2 & 2,3 & 2,1 & 1,5 \\
\hline Hipertensión & 21,8 & 26,7 & 18,8 & 18,4 \\
\hline
\end{tabular}

Los datos representan las medias y las desviaciones estándar o porcentajes.

* La fecha de cese del tabaquismo era desconocida para 134 participantes. 
Almudena Sánchez-Villegas, Alfredo Gea, Francisca Lahortiga-Ramos, Julio Martínez-González, Patricio Molero, Miguel A. Martínez-González

Tabla 2. Asociación (HR e IC 95\%) entre tabaquismo y riesgo de depresión en el estudio de cohorte SUN.

\begin{tabular}{|c|c|c|c|c|}
\hline & Casos & Persona-años & Modelo 1 & Modelo 2 \\
\hline Nunca ha sido fumador & 407 & 85610 & 1 (ref.) & 1 (ref.) \\
\hline Exfumador & 252 & 50757 & $1,08(0,92-1,27)$ & $1,05(0,89-1,25)$ \\
\hline Fumador actual & 230 & 38386 & $1,27(1,08-1,49)$ & $1,24(1,05-1,46)$ \\
\hline Nunca ha sido fumador & 407 & 85610 & 1 (ref.) & 1 (ref.) \\
\hline Exfumador >10a* & 86 & 20317 & $0,93(0,72-1,19)$ & $0,92(0,71-1,19)$ \\
\hline Exfumador $<10 a^{\star}$ & 161 & 29150 & $1,17(0,97-1,41)$ & $1,14(0,94-1,37)$ \\
\hline Fumador actual & 230 & 38386 & $1,27(1,08-1,49)$ & $1,24(1,05-1,47)$ \\
\hline \multicolumn{5}{|l|}{ Número de paquetes-año** } \\
\hline 0 & 407 & 85610 & 1 (ref.) & 1 (ref.) \\
\hline$\ll 10$ & 215 & 40741 & $1,07(0,91-1,27)$ & $1,08(0,91-1,28)$ \\
\hline $10-<20$ & 95 & 18453 & $1,15(0,91-1,44)$ & $1,11(0,88-1,40)$ \\
\hline $20+$ & 90 & 15056 & $1,50(1,16-1,93)$ & $1,38(1,07-1,79)$ \\
\hline Para +10 paquetes-año & & & $1,11(1,03-1,19)$ & $1,08(1,01-1,06)$ \\
\hline \multicolumn{5}{|c|}{ Cese del tabaquismo $(0 \text { a } 4-a)^{\star \star \star \star}$} \\
\hline No & 64 & 17018 & 1 (ref.) & 1 (ref.) \\
\hline Sí & 27 & 10653 & $0,65(0,41-1,02)$ & $0,63(0,40-0,99)$ \\
\hline
\end{tabular}

* La fecha de cese del tabaquismo no estuvo disponible en 134 participantes.

**Los datos sobre paquetes-año no estuvieron disponibles en 1423 participantes.

***Incluyendo solo a fumadores al inicio del estudio con datos durante 4 años de seguimiento y sin caso incidente de depresión durante los primeros 4 años de seguimiento $(n=2193)$.

HR: Hazard Ratio; IC: Intervalo de Confianza.

Modelo 1: HR e IC $95 \%$ ajustados por edad y sexo.

Modelo 2: El modelo 1 además ajustado por años de estudios (y, continuo), vivir solo/a (sí, no, desconocido), desempleado/a (sí/no), índice de masa corporal (kg/ $\mathrm{m}^{2}$, continuo), actividad física (METs-h/w, continuo), ingesta total de energía (kcal/d, continuo), ingesta de alcohol (cuatro categorías), adherencia a la dieta medite rránea (unidades del cuestionario MEDAS, continuo) y rasgos de personalidad (competitividad, tensión psicológica y dependencia: baja, media, alta, desconocida).

ximadamente 8-10 años. Estos resultados parecen consistentes con los resultados encontrados para la exposición al inicio del estudio. Por cada 47 participantes que dejaron de fumar, se pudo evitar 1 caso incidente durante los siguientes 10 años: NNT (IC 95\%): 47 (22-276). (Figura 3).

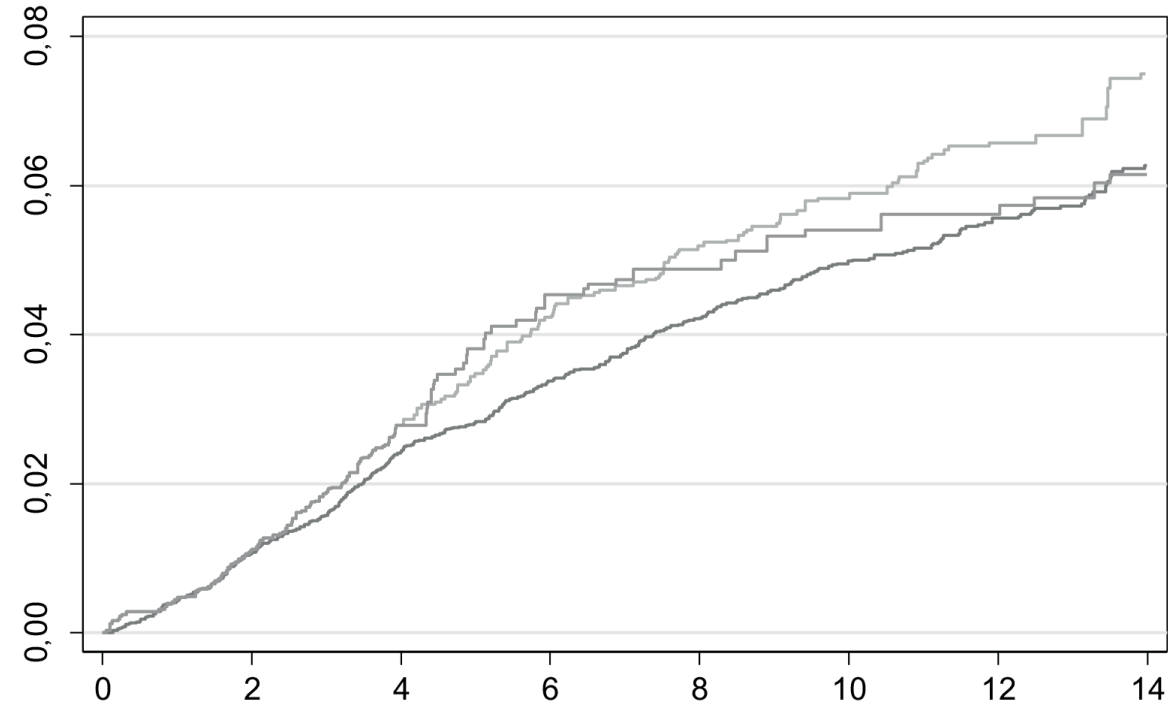

Seguimiento (años)

Nunca habían fumado Fumador/a — Fumador/a-Cese del tabaquismo

Figura 3. Incidencia acumulada de depresión según tabaquismo: Estudio de cohorte SUN 1999-2018. 
No se encontró interacción significativa alguna entre el consumo de tabaco y varias características de los participantes al inicio del estudio (sexo, edad, actividad física, adherencia a la dieta mediterránea o rasgos de personalidad) (Tabla 3). No obstante, los resultados sugirieron que fumar podría ser especialmente perjudicial entre los participantes más jóvenes con peores hábitos alimentarios y aquellos con varias características de personalidad (personas menos competitivas y más relajadas).

Los principales resultados no cambiaron después de realizar varios análisis de sensibilidad (Tabla 4).
Por último, para evaluar el papel de la depresión en el inicio del tabaquismo, los que nunca habían fumado y los exfumadores fueron evaluados después de 4 años de seguimiento con la presencia de depresión al inicio del estudio. Entre los 11.246 participantes con cuatro años de seguimiento y que nunca habían fumado al inicio del estudio, observamos un incremento significativo en el riesgo de inicio del tabaquismo asociado con la presencia de depresión al inicio del estudio (OR multivariable = 1,44; IC 95\% = 1,13-1,83). Cuando se repitió el análisis separando los que nunca habían fumado y los exfumadores, la asociación entre la presencia de

Tabla 3. Asociación (HR e IC 95\%) entre tabaquismo y riesgo de depresión en el estudio de cohorte SUN, según diversas características de los participantes.

\begin{tabular}{|c|c|c|c|c|c|c|c|}
\hline & $\mathbf{n}$ & Casos & $\begin{array}{l}\text { Nunca ha sido } \\
\text { fumador }\end{array}$ & Exfumador $>10 a^{*}$ & Exfumador $<10 a^{*}$ & Fumador actual & interacción $p$ \\
\hline Sexo & & & & & & & 0,319 \\
\hline Hombres & 6723 & 260 & 1 (ref.) & $1,09(0,72-1,64)$ & $0,97(0,67-1,41)$ & $1,35(0,98-1,84)$ & \\
\hline Mujer & 9662 & 624 & 1 (ref.) & $0,79(0,56-1,11)$ & $1,22(0,98-1,52)$ & $1,21(0,99-1,47)$ & \\
\hline Edad & & & & & & & 0,717 \\
\hline$<50 a$ & 13477 & 749 & 1 (ref.) & $1,03(0,76-1,41)$ & $1,19(0,97-1,45)$ & $1,27(1,07-1,52)$ & \\
\hline$\geq 50 \mathrm{a}$ & 2908 & 135 & 1 (ref.) & $0,80(0,52-1,24)$ & $0,96(0,56-1,64)$ & $1,01(0,61-1,68)$ & \\
\hline Dieta mediterránea & & & & & & & 0,745 \\
\hline Baja (« 6 puntos) & 7135 & 444 & 1 (ref.) & $1,05(0,71-1,55)$ & $1,09(0,83-1,43)$ & $1,29(1,03-1,63)$ & \\
\hline Alta ( $\geq 6$ puntos) & 9250 & 440 & 1 (ref.) & $0,83(0,59-1,16)$ & $1,18(0,91-1,52)$ & $1,19(0,93-1,52)$ & \\
\hline Actividad física & & & & & & & 0,636 \\
\hline Baja (< P50) & 8185 & 484 & 1 (ref.) & $0,97(0,67-1,40)$ & $1,26(0,98-1,62)$ & $1,24(0,99-1,54)$ & \\
\hline Alta $(\geq P 50)$ & 8200 & 400 & 1 (ref.) & $0,86(0,61-1,23)$ & $1,00(0,75-1,33)$ & $1,23(0,95-1,59)$ & \\
\hline Competitividad** & & & & & & & 0,510 \\
\hline Baja (< P50) & 9245 & 490 & 1 (ref.) & $0,94(0,66-1,34)$ & $1,13(0,88-1,46)$ & $1,39(1,12-1,73)$ & \\
\hline Alta ( $\geq$ P50) & 7283 & 399 & 1 (ref.) & $0,90(0,62-1,29)$ & $1,14(0,87-1,50)$ & $1,06(0,82-1,38)$ & \\
\hline Tensión** & & & & & & & 0,272 \\
\hline Baja (८ P50) & 9133 & 383 & 1 (ref.) & $0,91(0,60-1,39)$ & $1,41(1,06-1,87)$ & $1,42(1,11-1,81)$ & \\
\hline Alta ( $\geq$ P50) & 7403 & 509 & 1 (ref.) & $0,89(0,65-1,23)$ & $0,97(0,76-1,24)$ & $1,13(0,90-1,41)$ & \\
\hline Dependencia** & & & & & & & 0,408 \\
\hline Baja (८ P50) & 10147 & 503 & 1 (ref.) & $0,96(0,69-1,34)$ & $1,29(1,01-1,65)$ & $1,24(0,99-1,55)$ & \\
\hline Alta $(\geq \mathrm{P} 50)$ & 6469 & 391 & 1 (ref.) & $0,84(0,56-1,26)$ & $0,95(0,71-1,26)$ & $1,22(0,95-1,56)$ & \\
\hline
\end{tabular}

* La fecha de cese del tabaquismo no estuvo disponible en 134 participantes.

**Varios valores ausentes para competitividad, tensión y dependencia.

HR: Hazard Ratio; IC: Intervalo de Confianza; P50: Percentil 50.

Tabla 4. Asociación (HR e IC 95\%) entre tabaquismo y riesgo de depresión en el estudio de cohorte SUN. Análisis de sensibilidad.

\begin{tabular}{|c|c|c|c|c|}
\hline & casos/N & Exfumador $>10 a^{\star}$ & Exfumador $<10 a^{*}$ & Fumador actual \\
\hline Muestra general & $884 / 16385$ & $0,92(0,71-1,19)$ & $1,14(0,94-1,37)$ & $1,24(1,05-1,47)$ \\
\hline $\begin{array}{l}\text { Análisis desde el inicio del estudio hasta los primeros } 6 \text { años de } \\
\text { seguimiento }\end{array}$ & $545 / 16385$ & $0,78(0,56-1,09)$ & $1,09(0,86-1,39)$ & $1,30(1,05-1,60)$ \\
\hline $\begin{array}{l}\text { Considerando solo los casos diagnosticados después de los primeros } 2 \\
\text { años de seguimiento }\end{array}$ & $716 / 16138$ & $1,01(0,76-1,34)$ & $1,18(0,96-1,45)$ & $1,27(1,05-1,53)$ \\
\hline $\begin{array}{l}\text { Considerando solo los casos diagnosticados después de los primeros } 4 \\
\text { años de seguimiento }\end{array}$ & $488 / 14900$ & $1,03(0,73-1,46)$ & $1,15(0,89-1,48)$ & $1,29(1,04-1,62)$ \\
\hline $\begin{array}{l}\text { Excluyendo a los participantes con enfermedad cardiovascular, diabetes } \\
\text { o cáncer al inicio del estudio }\end{array}$ & $827 / 15423$ & $1,02(0,78-1,32)$ & $1,11(0,92-1,35)$ & $1,25(1,05-1,49)$ \\
\hline
\end{tabular}

* La fecha de cese del tabaquismo no estuvo disponible en 134 participantes.

Ajustado por sexo, edad, años de estudios (y, continuo), vivir solo/a (sí, no, desconocido), desempleado (sí/no), índice de masa corporal (kg/m2, continuo), actividad física (METs-h/w, continuo), ingesta total de energía (kcal/d, continuo), ingesta de alcohol (cuatro categorías), adherencia a la dieta mediterránea (unidades del cuestionario MEDAS, continuo) y rasgos de personalidad (competitividad, tensión psicológica y dependencia: baja, media, alta, desconocida).

HR: Hazard Ratio; IC: Intervalo de Confianza.; Q 2: cuestionario 2; 2-a de seguimiento; Q 4: cuestionario 4; 4-a de seguimiento. 
depresión al inicio del estudio y el riesgo de inicio/recaída del tabaquismo dejó de ser significativa. El OR multivariable para el inicio del tabaquismo entre los que nunca habían fumado: $1,13(0,71-1,82)$, y el OR para la recaída del tabaquismo entre los exfumadores: 1,26 (0,89-1,78).

\section{Discusión}

En estos análisis realizados en el marco de la cohorte SUN, encontramos que ser fumador actual tenía una asociación posible con un mayor riesgo de depresión. Además, los participantes con altas dosis de consumo de tabaco y duración del tabaquismo (medido como al menos 20 paquetes-año de consumo) tuvieron un aumento significativo en el riesgo de desarrollar depresión durante el seguimiento. Es más, el cese del tabaquismo tuvo una asociación inversa con la incidencia de depresión.

Estos resultados confirman los resultados obtenidos en nuestra cohorte hace más de 10 años con un menor tiempo de seguimiento y un menor tamaño muestral. En ese análisis, ser fumador se asoció con un incremento relativo de $39 \%$ en el riesgo de depresión. Además, la duración del tabaquismo y el número de cigarrillos fumados por día también estuvieron asociados al riesgo de depresión (Sánchez-Villegas, Serrano-Martínez, Alonso, Tortosa y Martínez-González, 2008b).

En nuestra cohorte, la presencia de depresión al inicio del estudio no parece tener asociación con el inicio del tabaquismo. Sin embargo, aunque la asociación no era estadísticamente significativa para la recaída del tabaquismo entre exfumadores, la magnitud de la asociación podría considerarse moderada, con un incremento de $26 \%$ en el riesgo de recaída del tabaquismo. Es probable que el pequeño número de participantes en este análisis no nos permitió detectar asociaciones estadísticamente significativas.

Algunos estudios longitudinales han informado de un papel perjudicial del tabaquismo en la depresión (Clyde, Smith, Gariépy y Schmitz, 2015; Flensborg-Madsen et al., 2011; Raffetti, Donato, Forsell y Galanti, 2019; Zhang, Woud, Becker y Margraf, 2018) aunque los resultados no son totalmente consistentes (Bruin, Comijs, Kok, Van der Mast y Van den Berg, 2018; Furihata et al., 2018).

Varios mecanismos podrían explicar el papel perjudicial del tabaco en la depresión. La exposición a la nicotina y otros aditivos de los cigarrillos de tabaco podría predisponer a la depresión a través de varios mecanismos fisiológicos relacionados con la función del sistema nervioso central. Por ejemplo, los fumadores muestran una reducción importante en los niveles de monoamino oxidasa B (Fowler et al., 1996). Esta enzima está implicada en el catabolismo oxidativo cerebral y periférico de los neurotransmisores, incluyendo la dopamina. La desregulación del sistema dopamínico se ha asociado recientemente con varios síntomas centrales en la depresión, como la presencia de anhedonia (Belujon y
Grace, 2017). Es más, los modelos animales y los estudios en humanos han sugerido que la exposición a largo plazo a la nicotina podría actuar como un factor de estrés que resulta en una desregulación del eje hipotalámico-pituitario-adrenal (HPA) (Furihata et al, 2018) con la elevación simultánea del cortisol, la hormona adrenocorticotrópica y la hormona liberadora de corticotropina. Esta desregulación estaría implicada en un volumen reducido del hipocampo, tasas reducidas de neurogénesis y niveles aumentados de citocinas proinflamatorias y marcadores de estrés oxidativo. Tanto la inflamación sistémica de bajo grado como el estrés oxidativo están implicados en varios mecanismos fisiopatológicos relacionados con la depresión.

El papel del tabaquismo en el riesgo de depresión podría estar mediado por la incidencia de varias enfermedades resultantes del tabaquismo, como la enfermedad cardiovascular que se ha asociado con la depresión en nuestra cohorte (Molero et al., 2017).

Otra posible explicación de la asociación entre el tabaquismo y la depresión es la presencia de varios factores de confusión asociadas de forma independiente con ambas variables, tales como las características de estilo de vida o de personalidad. Sin embargo, esta explicación es poco probable ya que hemos ajustado los modelos para varias variables de estilo de vida (p. ej., actividad física, ingesta de alcohol o hábitos alimentarios) y rasgos de personalidad tales como tensión, competitividad o nivel de dependencia. Por último, los participantes con depresión subclínica, pero sin un diagnóstico de depresión al inicio del estudio, podrían haber iniciado el hábito de fumar para aliviar sus síntomas iniciales de una depresión no diagnosticada al inicio del estudio y los resultados obtenidos podrían haber sido una consecuencia de un sesgo de causalidad inversa. Es más probable que estos casos no diagnosticados potencialmente responsables de un sesgo de causalidad inversa se informen después de los primeros 2-4 años de seguimiento. Sin embargo, los resultados no cambiaron cuando repetimos los análisis después de excluir los casos tempranos (en los primeros 2 o 4 años de seguimiento).

La presencia de depresión al inicio del estudio fue también prospectivamente asociada con una probabilidad más alta de inicio de tabaquismo en nuestra cohorte, lo que sugiere una asociación bidireccional entre el tabaquismo y la depresión. Algunos otros estudios han informado resultados del mismo razonamiento. Una muestra representativa de 44.921 adolescentes encontró que, entre los no fumadores, la prevalencia de depresión a lo largo de la vida era aproximadamente $50 \%$ menor que entre los fumadores (Cohn, 2018). Otros estudios han encontrado que entre los fumadores con enfermedades mentales las tasas de cese del tabaquismo siguen siendo consistentemente más bajas que entre los fumadores sin síntomas depresivos (Huffman, Bromberg y Augustson, 2018; Secades-Villa, Weidberg, González-Roz, Reed y Fernández-Hermida, 2018). Una de las explicacio- 
nes más aceptadas es la basada en la hipótesis de la automedicación (ya mencionada) que propone que las personas recurren al tabaquismo para aliviar sus síntomas pero las empeoran con el tiempo (Fluharty et al., 2017). Otra posible explicación de esta asociación bidireccional es la vulnerabilidad compartida tanto para la adicción al tabaco como para la aparición de depresión, aunque eso no apoyaría una reducción del riesgo de depresión asociada con el cese del tabaquismo en este y otros estudios (Khaled et al., 2012; Zarghami, Taghizadeh, Sharifpour y Alipour, 2018).

Deben ser mencionadas las fortalezas de este estudio, que incluyen su diseño prospectivo longitudinal, el uso de métodos previamente validados, el gran tamaño muestral y el extenso control de los factores de confusión, incluyendo factores alimentarios. Los participantes tenían un alto nivel de estudios, lo que aumenta la calidad de la información autoinformada y reduce la posibilidad de sesgo de clasificación errónea. Además, la restricción a un subgrupo bastante homogéneo de participantes respecto del nivel de estudios minimiza la posibilidad de confusión residual y es una técnica excelente para mejorar la validez interna de nuestros resultados.

Sin embargo, algunas posibles limitaciones también deben abordarse. Una limitación de este estudio es que el diagnóstico de depresión fue autoinformado, aunque la validez del enfoque adoptado para adjudicar casos incidentes de depresión durante el seguimiento se validó en una submuestra de esta cohorte (Sánchez-Villegas et al., 2008a). Además de una cohorte, el diseño mitiga esto hasta cierto punto. Restringimos nuestra cohorte a participantes con un alto nivel de estudios para obtener una mejor calidad de información autoinformada. En este contexto, es más probable que la clasificación errónea no sea diferencial y, por tanto, sesgaría los resultados hacia el nulo.

Aunque todos los resultados se ajustaron para una variedad de posibles factores de confusión importantes, no podemos excluir la posibilidad de algunos factores desconocidos o no medidos que podrían dar una explicación parcial de los resultados informados. Esta posibilidad encajará bien en la hipótesis de vulnerabilidad compartida y admitimos que esta limitación es inherente al diseño observacional de nuestro estudio. Sin embargo, sería poco probable que dicho factor desconocido que resulte en una «vulnerabilidad compartida» no se correlacione con los muchos posibles factores de confusión controlados en nuestro estudio. Por último, nuestra muestra no fue representativa de la población general. Sin embargo, la falta de representatividad no impide el establecimiento de asociaciones que puedan generalizarse a otros grupos, siempre que ningún mecanismo biológico sugiera que la asociación sea inválida para otras poblaciones. Ha sido recomendada la selección de grupos de estudio que mantienen la homogeneidad respecto de importantes factores de confusión como el nivel de estudios para maximizar la validez de cohortes, en lugar de pretender obtener una «muestra representativa» de una población natural (Rothman, Greenland y Lash, 2008), especialmente en el estudio de conductas altamente complejas como las que median el trastorno depresivo y el tabaquismo.

En conclusión, en el estudio de cohorte SUN se encontró una asociación bidireccional entre el tabaquismo y la depresión, lo que refuerza las conclusiones de estudios previos. La novedad aportada por nuestro estudio yace en el uso de un diseño prospectivo longitudinal y el análisis simultáneo del uso, la dosis y la duración del tabaquismo. También evaluamos el papel del cese del tabaquismo en el riesgo de depresión y encontramos que el cese del tabaquismo tuvo una asociación inversa con la incidencia de depresión. Es más, la presencia de un trastorno depresivo se asoció prospectivamente con el inicio del tabaquismo, lo que sugiere una asociación bidireccional entre el tabaquismo y la depresión. Por tanto, el control del tabaquismo y las campañas de promoción de la salud para dejar de fumar podrían considerarse estrategias efectivas de salud pública para prevenir y gestionar los trastornos depresivos.

\section{Reconocimientos}

Los autores desean expresar su gratitud al personal, los participantes y otros investigadores del proyecto SUN por su valioso apoyo y colaboración. El proyecto SUN recibió financiación del Instituto de Salud Carlos III-Gobierno de España y el Fondo europeo de desarrollo regional-Unión Europea (RD 06/0045, CIBER-OBN, Ayudas PI14/01668, PI14/01798, PI14/01764, PI17/01795 y G03/140) y la Universidad de Navarra.

También agradecemos la colaboración de otros miembros del grupo SUN: Alonso A, Álvarez-Álvarez I, Balaguer A, Barbagallo M, Barrientos I, Barrio-López MT, Basterra-Gortari FJ, Battezzati A, Bazal P, Benito S, Bertoli S, Bes-Rastrollo M, Beulen Y, Beunza JJ, Buil-Cosiales P, Canales M, Carlos S, Carmona L, Cervantes S, Cristobo C, de Irala J, de la Fuente-Arrillaga C, de la O V, de la Rosa PA, Delgado-Rodríguez M, Díaz-Gutiérrez J, Díez Espino J, Domínguez L, Donat-Vargas C, Donazar M, Eguaras S, Fresán U, Galbete C, García-Arellano A, García López M, Gardeazábal I, Gea A, GutiérrezBedmar M, Gomes-Domingos AL, Gómez-Donoso C, Gómez-Gracia E, Goñi E, Goñi L, Guillén F, Henríquez P, Hernández-Hernández A, Hershey MS, Hidalgo-Santamaría M, Hu E, Leone A, Llorca J, López del Burgo C, Marí A, Marques I, Martí A, Martín Calvo N, Martín-Moreno JM, Martínez JA, Martínez-Lapiscina EH, Mendonça R, Menéndez C, Molendijk M, Murphy K, Muñoz M, Núñez-Córdoba JM, Pajares R, Papadaki A, Parletta N, Pérez de Ciriza P, Pérez-Cornago A, Pérez de Rojas J, Pimenta AM, Pons J, Ramallal R, Razquin C, Rico-Campà A, Romanos A, Ruano C, Ruiz-Estigarribia L, Ruiz Zambrana A, Salgado E, San Julián B, Sánchez D, Sánchez-Bayona R, Sánchez-Tainta A,Santiago S, Sayón-Orea C, Schlatter J, 
Serrano-Martinez M, Toledo E, Toledo J, Tortosa A, Valencia F, Vázquez Z, Zarnowiecki D, Zazpe I. Todos contribuyeron a la creación de esta cohorte y la validación de los cuestionarios utilizados en este estudio.

\section{Conflicto de intereses}

Sin relevancia para este trabajo, PM informa de percibir ayuda de la Clínica Universidad de Navarra y haber recibido becas de investigación del Ministerio de Educación (España), el Gobierno de Navarra (España), la Fundación Española de Psiquiatría y Salud Mental y AstraZeneca; es consultor clínico de MedAvanteProPhase y ha recibido honorarios por conferencias o ha sido consultor de AB-Biotics, Janssen, Novumed, Roland Berger y Scienta. Los autores declaran la inexistencia de conflicto de intereses.

\section{Referencias}

Alonso, A., Beunza, J. J., Delgado-Rodríguez, M. y Martínez-González, M. A. (2005). Validation of self reported diagnosis of hypertension in a cohort of university graduates in Spain. BMC Public Health, 5, 94. doi:10.1186/14712458-5-94

Belujon, P. y Grace, A. A. (2017). Dopamine system dysregulation in major depressive disorders. International Journal of Neuropsychopharmacology, 20, 1036-1046. doi:10.1093/ ijnp/pyx056.

Bes-Rastrollo, M., Pérez-Valdivieso, J. R., Sánchez-Villegas, A., Alonso, Á. y Martínez-González, M. Á. (2005). Validation of the self-reported weight and body mass index of the participants in a cohort of university graduates. Revista Española de Obesidad, 3, 352-358.

Bruin, M. C., Comijs, H. C., Kok, R. M., Van der Mast, R. C. y Van den Berg, J. F. (2018). Lifestyle factors and the course of depression in older adults: A NESDO study. International Journal of Geriatric Psychiatry, 33, 1000-1008. doi:10.1002/gps.4889.

Carlos, S., De la Fuente-Arrillaga, C., Bes-Rastrollo, M., Razquin, C., Rico-Campà, A., Martínez-González, M. A. y Ruiz-Canela, M. (2018). Mediterranean diet and health outcomes in the SUN cohort. Nutrients, 10, 439. doi: $10.3390 /$ nu10040439.

Clyde, M., Smith, K. J., Gariépy, G. y Schmitz, N. (2015). Assessing the longitudinal associations and stability of smoking and depression syndrome over a 4-year period in a community sample with type 2 diabetes. Journal of Diabetes, 95-101. doi:10.1111/1753-0407.12141.

Cohn, A. M. (2018). Never, non-daily, and daily smoking status and progression to daily cigarette smoking as correlates of major depressive episode in a national sample of youth: Results from the national survey of drug use and health 2013 to 2015. Addictive Behaviors, 84, 118-125. doi:10.1016/j.addbeh.2018.03.001.
De la Fuente-Arrillaga, C., Vázquez Ruiz, Z., Bes-Rastrollo, M., Sampson, L. y Martínez-González, M. A. (2010). Reproducibility of an FFQ validated in Spain. Public Health Nutrition, 13, 1364-1372. doi:10.1017/S1368980009993065.

Fernández-Ballart, J. D., Piñol, J. L., Zazpe, I., Corella, D., Carrasco, P., Toledo, E.,... Martín-Moreno, J. M. (2010). Relative validity of a semi-quantitative food-frequency questionnaire in an elderly Mediterranean population of Spain. British Journal of Nutrition, 103, 1808-1816. doi:10.1017/S0007114509993837.

Fernández-Montero, A., Moreno-Galarraga, L., Sánchez-Villegas, A., Lahortiga-Ramos, F., Ruiz-Canela, M., Martínez-González, M. Á. y Molero, P. (2020). Dimensions of leisure-time physical activity and risk of depression in the "Seguimiento Universidad de Navarra" (SUN) prospective cohort. BMC Psychiatry, 20, 1-9. doi:10.1186/s12888020-02502-6.

Flensborg-Madsen, T., von Scholten, M. B., Flachs, E. M., Mortensen, E. L., Prescott, E. y Tolstrup, J. S. (2011). Tobacco smoking as a risk factor for depression. A 26-year population-based follow-up study. Journal of Psychiatric Research, 45, 143-149. doi:10.1016/j.jpsychires.2010.06.006.

Fluharty, M. E., Taylor, A. E., Grabski, M. y Munafò, M. R. (2017). The association of cigarette smoking with depression and anxiety: A systematic review. Nicotine $\mathcal{E}$ Tobacco Research, 19, 3-13. doi:10.1093/ntr/ntw140.

Fluharty, M. E., Sallis, H. y Munafò, M. R. (2018). Investigating possible causal effects of externalizing behaviors on tobacco initiation: A Mendelian randomization analysis. Drug and Alcohol Dependence, 191, 338-342.

Fowler, J. S., Volkow, N. D., Wang, G. J., Pappas, N., Logan, J., MacGregor, R.,... Cilento, R. (1996). Inhibition of monoamine oxidase B in the brains of smokers. Nature, 379, 733-736. doi:10.1038/379733a0.

Furihata, R., Konno, C., Suzuki, M., Takahashi, S., Kaneita, Y., Ohida, T. y Uchiyama, M. (2018). Unhealthy lifestyle factors and depressive symptoms: A Japanese general adult population survey. Journal of Affective Disorders, 234, 156-161. doi:10.1016/j.jad.2018.02.093.

Huffman, A. L., Bromberg, J. E. y Augustson, E. M. (2018). Lifetime depression, other mental illness, and smoking cessation. American Journal of Health Behavior, 42, 90-101. doi:10.5993/AJHB.42.4.9.

Instituto Nacional del Cáncer [National Cancer Institute] (2019). Dictionary of cancer terms. Recuperado de https://www.cancer.gov/publications/dictionaries/cancer-terms/def/pack-year.

James, S. L., Abate, D., Abate, K. H., Abay, S. M., Abbafati, C., Abbasi, N.,... Murray, C. J. L. (2018). Global, regional, and national incidence, prevalence, and years lived with disability for 354 diseases and injuries for 195 countries and territories, 1990-2017: A systematic analysis for the global burden of disease study 2017. Lancet, 392, 17891858. doi:10.1016/S0140-6736(18)32279-7. 
Jiménez-Treviño, L., Velasco, A., Rodríguez-Revuelta, J., Abad, I., De la Fuente-Tomás, L., González-Blanco, L.,... Sáiz, P. A. (2019). Factores asociados con consumo de tabaco en pacientes con depresión. Adicciones, 31, 298-308. doi:10.20882/adicciones.1191.

Kedzior, K. K. y Laeber, L. T. (2014). A positive association between anxiety disorders and cannabis use or cannabis use disorders in the general population-a meta-analysis of 31 studies. BMC Psychiatry, 14, 1-22. doi:10.1186/1471244X-14-136.

Khaled, S. M., Bulloch, A. G., Williams, J. V., Hill, J. C., Lavorato, D. H. y Patten, S. B. (2012). Persistent heavy smoking as risk factor for major depression (MD) incidenceevidence from a longitudinal Canadian cohort of the national population health survey. Journal of Psychiatric Research, 46, 436-443. doi:10.1016/j.jpsychires.2011.11.011.

Kyu, H. H., Abate, D., Abate, K. H., Abay, S. M., Abbafati, C., Abbasi, N.,... Murray, C. J. L. (2018). Global, regional, and national disability-adjusted life-years (DALYs) for 359 diseases and injuries and healthy life expectancy (HALE) for 195 countries and territories, 1990-2017: A systematic analysis for the global burden of disease study 2017. Lancet, 392, 1859-1922. doi:10.1016/S0140-6736(18)32335-3.

Lassale, C., Batty, G. D., Baghdadli, A., Jacka, F., Sánchez-Villegas, A., Kivimäki, M. y Akbaraly, T. (2019). Healthy dietary indices and risk of depressive outcomes: A systematic review and meta-analysis of observational studies. Molecular Psychiatry, 24, 965-986. doi:10.1038/s41380-0180237-8.

Martínez-González, M. A., Sánchez-Villegas, A., De Irala, J., Marti, A. y Martínez, J. A. (2002). Mediterranean diet and stroke: Objectivesand design of the SUN project. Nutritional Neuroscience, 5, 65-73. doi:10.1080/10284150290007047.

Martínez-González, M. A., López-Fontana, C., Varo, J. J., Sánchez-Villegas, A. y Martínez, J. A. (2005). Validation of the Spanish version of the physical activity questionnaire used in the nurses' health study and the health professionals' follow-up study. Public Health Nutrition, 8, 920-927. doi:10.1079/phn2005745.

Mataix, J. (2003). Tabla de composición de alimentos (Food Composition Tables). Granada: University of Granada.

Molero, P., Martínez-González, M. Á., Ruiz-Canela, M., Lahortiga, F., Sánchez-Villegas, A., Pérez-Cornago, A. y Gea, A. (2017). Cardiovascular risk and incidence of depression in young and older adults: Evidence from the SUN cohort study. World Psychiatry, 16, 111. doi:10.1002/ wps. 20390.

Moreiras, O., Carbajal, A., Cabrera, L. y Cuadrado, C. (2005). Tablas de composición de alimentos (Food Composition Tables). Madrid: Ediciones Pirámide.

Opie, R. S., Itsiopoulos, C., Parletta, N., Sánchez-Villegas, A., Akbaraly, T. N., Ruusunen, A. y Jacka, F. N. (2017). Dietary recommendations for the prevention of depression.
Nutritional Neuroscience, 20, 161-171. doi:10.1179/1476830 515Y.0000000043.

Raffetti, E., Donato, F., Forsell, Y. y Galanti, M. R. (2019). Longitudinal association between tobacco use and the onset of depressive symptoms among Swedish adolescents: The Kupol cohort study. European Child Eं Adolescent Psychiatry, 28, 695-704. doi:10.1007/s00787-018-1237-6.

Rothman, K. J., Greenland, S. y Lash, T. L. (Eds.) (2008). Modern epidemiology ( $3^{\text {rd }}$ ed). Philadelphia: Lippincott Williams \& Wilkins.

Sánchez-Villegas, A., Schlatter, J., Ortuno, F., Lahortiga, F., Pla, J., Benito, S. y Martínez-González, M. A. (2008a). Validity of a self-reported diagnosis of depression among participants in a cohort study using the Structured Clinical Interview for DSM-IV (SCID-I). BMC Psychiatry, 8, 1-8. doi:10.1186/1471-244X-8-43.

Sánchez-Villegas, A., Serrano-Martínez, M., Alonso, A., Tortosa, A. y Martínez-González, M. Á. (2008b). Role of tobacco use on the depression incidence in the SUN cohort study. Medicina Clínica, 130, 405-409. doi:10.1157/13117850.

Schröder, H., Fitó, M., Estruch, R., Martínez-González, M. A., Corella, D., Salas-Salvadó, J.,... Covas, M. I. (2011). A short screener is valid for assessing Mediterranean diet adherence among older Spanish men and women. The Journal of Nutrition, 141, 1140-1145. doi:10.3945/ jn.110.135566.

Secades-Villa, R., Weidberg, S., González-Roz, A., Reed, D. D. y Fernández-Hermida, J. R. (2018). Cigarette demand among smokers with elevated depressive symptoms: An experimental comparison with low depressive symptoms. Psychopharmacology, 235, 719-728. doi:10.1007/s00213017-4788-1.

Taylor, G., McNeill, A., Girling, A., Farley, A., Lindson-Hawley, N. y Aveyard, P. (2014). Change in mental health after smoking cessation: Systematic review and meta-analysis. BMJ, 348. doi:10.1136/bmj.g1151.

Zarghami, M., Taghizadeh, F., Sharifpour, A. y Alipour, A. (2018). Efficacy of smoking cessation on stress, anxiety, and depression in smokers with chronic obstructive pulmonary disease: A randomized controlled clinical trial. Addiction E⿱ health, 10, 137. doi:10.22122/ahj.v10i3.600.

Zhang, X. C., Woud, M. L., Becker, E. S. y Margraf, J. (2018). Do health-related factors predict major depression? A longitudinal epidemiologic study. Clinical Psychology $\mathcal{E}^{\circ}$ Psychotherapy, 25, 378-387. doi:10.1002/cpp.2171. 